\title{
Género, movilidad y frontera. La identidad de las mujeres portuguesas que emigraron a Canarias durante la Unión Ibérica
}

\section{Gender, migration and border. The identity of portuguese women who emigrated to the Canary Islands during the Iberian Union}

\author{
Javier Luis Álvarez Santos* \\ https://orcid.org/0000-0002-7845-6521
}

\begin{abstract}
Resumo
Ao longo do último quartel do século XVI, especialmente após a agregação da Coroa portuguesa à Monarquia Hispânica, a consolidação do mundo atlântico deu-se por meio de complexas redes socioeconômicas transoceânicas. Nesse cenário, as Ilhas Canárias tornaram-se locais atrativos para a realização de novas atividades profissionais e comerciais. A comunidade portuguesa que se instalou nas ilhas durante este período favoreceu a internacionalização destes territórios ao incluí-los nas suas redes atlânticas. No entanto, a agência das mulheres portuguesas que emigraram para o Arquipélago ainda é desconhecida. 0 objetivo desta investigação, com base na análise de fontes documentais primárias, é identificar as mulheres portuguesas que se fixaram nas ilhas, conhecer as relações locais e transnacionais em que se encontram inseridas e, em última instância, caracterizar a identidade destes sujeitos com base no gênero e na mobilidade transfronteiriça.
\end{abstract}

Palavras-chave: gênero, Português, Canárias, mobilidade, identidade.

\begin{abstract}
The consolidation of the Atlantic world is gestated from complex transoceanic socioeconomic networks throughout the last quarter of the 16th century, especially after the addition of the Portuguese Crown to the Hispanic Monarchy. In this context, the Canary Islands became attractive places to undertake new professional and commercial activities. The Portuguese community that settled on the islands during this period favoured the internationalization of these territories by including them in their Atlantic networks. However, the agency of the Portuguese women who migrated to the Archipelago is still unknown. From the analysis of primary documentary sources, the objective of this investigation is to identify the Portuguese women who settled in the
\end{abstract}

\footnotetext{
* Doutor em História Moderna pela Universidade Nova de Lisboa e a Universidad de La Laguna. Investigador júnior do CHAM-Centro de Humanidades, Faculdade de Ciências Sociais e Humanas, FCSH, Universidade Nova de Lisboa.E-mail: jlasantos@fcsh.unl.pt
} 
Islands, to know the local and transnational relationships in which they are inserted and, ultimately, to characterize the identity of these subjects based on gender and cross-border mobility.

Keywords: gender, Portuguese, Canary Islands, mobility, identity.

\section{Introducción}

Especial significancia tuvo la comunidad portuguesa en Canarias a lo largo del último cuarto del siglo XVI, en especial tras la agregación de la Corona portuguesa a la Monarquía Hispánica. En primer lugar, porque superaron ampliamente en número a cualquier otra comunidad forastera establecida en el Archipiélago hasta finales del siglo XVII. En segundo lugar, por la capacidad de adaptación y de integración en la sociedad de acogida hasta alcanzar la mimetización o insularización. En tercer lugar, porque los emigrantes portugueses en las Islas favorecieron la internacionalización del territorio isleño al incluir estos espacios en las redes transcontinentales de las que formaban parte.

Coincide esta época con el periodo de consolidación del mundo atlántico a partir de complejas redes mercantiles transoceánicas. Al mismo tiempo, en Canarias se produce un cambio de ciclo económico, en el que se sustituye el principal cultivo exportador de las Islas: el azúcar por la vid. El auge del comercio del vino canario supuso que estos peñascos en el Océano se convirtieran en lugares atractivos para emprender y desarrollar nuevas actividades profesionales y mercantiles. Paralelamente, las Islas, alejadas de su metrópoli, concibieron lazos de complementariedad económica con los archipiélagos portugueses de la Macaronesia para asegurar su subsistencia y forjaron relaciones mercantiles con el entorno colonial americano y africano para garantizar el abastecimiento de productos exógenos y afianzar el comercio de productos insulares en mercados foráneos. De este modo, estos espacios insulares se insertaron en su entorno circundante más inmediato: el Atlántico. En este contexto, la sociedad insular facilitó y tolero la llegada de foráneos que pusiesen en explotación y en valor las tierras y sus cultivos y, sobre todo, que conectasen estos territorios en el seno del Océano con los espacios continentales.

Sin embargo, aún es poco conocida la agencia de las mujeres portuguesas emigrantes durante este periodo de consolidación del mundo atlántico. ¿Qué 
caracterizó a estas mujeres que emigraron hacia territorios coloniales castellanos en el seno del Océano? ¿Cómo se definió su personalidad en el lugar de acogida? ¿Cuál fue su desempeño en la sociedad insular y en las redes familiares transfronterizas? ¿Qué implicaciones tuvo para este grupo su triple condición de mujeres, emigrantes y forasteras en un espacio de frontera cuya identidad aún estaba en construcción?

Precisamente, este estudio aborda esta problemática con el propósito de reconocer la huella de las mujeres portuguesas que llegaron y se establecieron en las Islas; discernir las relaciones sociales, económicas y profesionales en las que se insertan, tanto con los insulares como con la comunidad de origen; y caracterizarlas como sujetos activos en la dinamización de las Islas. En definitiva, estos objetivos tienen como finalidad caracterizar la propia identidad de las mujeres emigrantes procedentes de Portugal en Canarias a partir de su dinamismo en el proceso de consolidación del mundo atlántico desde la agregación de la Corona portuguesa a la Monarquía Hispánica.

Metodológicamente se sustenta esta investigación en el análisis de fuentes documentales primarias insulares. A este respecto, por un lado, hemos abordado documentación inédita, principalmente notarial y eclesiástica. Hemos estudiado la Sección Histórica de Protocolos Notariales del Archivo Histórico Provincial de Santa Cruz de Tenerife a través de catas por escribanías y años y hemos consultado las "Informaciones de Solterías y Viudedades, procedentes de los fondos del Archivo Histórico Diocesano de San Cristóbal de La Laguna y del Archivo Histórico Diocesano del Obispado de Canarias. Por otro lado, hemos recuperado y sistematizado las referencias a mujeres portuguesas en otras fuentes primarias que fueron publicadas por otros historiadores en el contexto de investigaciones cuyo objeto de estudio no eran las mujeres ${ }^{1}$. En este sentido, planteamos una relectura de las fuentes desde la perspectiva de género para construir una nueva narrativa histórica sobre las identidades en espacios de frontera y que ésta se inserte tanto en los análisis más innovadores sobre la historia regional como en los estudios atlánticos y transnacionales.

A este respecto, abordamos la huella de las mujeres forasteras, en particular la portuguesa, en la conformación de la sociedad insular sobre la base de la representación y la consideración que sobre ellas fundamentaron las fuentes primarias. En primer lugar, analizaremos las fuentes primarias y los datos cuantitativos extraídos de estos documentos con el fin de visibilizar

\footnotetext{
${ }^{1}$ Nos referimos a los trabajos publicados por la historiografía española que, a finales del siglo XX y principios del siglo XXI, abordaron la problemática de la formación de la sociedad canaria. En concreto, destacamos las aportaciones de Fajardo Spínola, Lobo Cabrera, Torres Santana y Brito González.
} 
y reconocer a estas mujeres emigrantes que pasaron por la Islas. En segundo lugar, a partir de esta información recogida, pasaremos a estudiar la agencia de estas mujeres lusas durante este periodo, tanto en el ámbito insular como en el contexto iberoatlántico.

\section{Género, emigración y frontera: un análisis historiográfico para repensar la identidad}

Los portugueses que emigraron a Canarias durante el periodo de la agregación portuguesa a la Monarquía Hispánica son un sujeto exógeno al Archipiélago que, como otras naciones foráneas, incorpora su bagaje sociocultural a una población recientemente formada y que aún está definiendo su propia identidad. No obstante, a diferencia de otras comunidades europeas, la perdurabilidad temporal de la población portuguesa en las Islas facilitó, primeramente, su integración y asimilación para, finalmente, asistir y contribuir en un proceso de simbiosis cultural y mimetismo social con la población local. ${ }^{2}$

Sobre esta conceptualización de las identidades, Baena Zapatero en su estudio sobre las mujeres criollas en Nueva España durante los siglos XVI y XVII se cuestiona «cómo es posible que la mitad de la población haya sido excluida del proceso de construcción de su propia identidad». ${ }^{3}$ Precisamente es esta misma disyuntiva la que hemos abordado en el presente trabajo para visibilizar e identificar a un conjunto poblacional específico.

El objeto de estudio al que nos hemos aproximado se ha abordado desde una doble perspectiva: origen y género. Por tanto, la metodología empleada en esta investigación debe tratar las particularidades del individuo a través de la intersección de estas dos singularidades. En este sentido, el método de análisis empleado examina al individuo desde los planteamientos de la interseccionalidad. Es decir, desde su triple condición de mujer, emigrante y forastera, con el objetivo de visibilizar un sujeto de estudio marginado en los márgenes del Imperio.

Señala Monzón Perdomo que el análisis del pasado de las mujeres es hoy más factible que nunca. En las últimas décadas del siglo XX y, sobre todo, desde los primeros años de la presente centuria, se han venido produciendo

${ }^{2}$ ÁLVAREZ SANTOS, Javier Luis. "Identidad y pacto social: los portugueses en Canarias durante la Unión Ibérica" In Anuario de Historia Regional y de las Fronteras. 2019, 24, pp. 139-154.

${ }^{3}$ BAENA ZAPATERO, Alberto. Mujeres novohispanas e identidad criollas. S. XVI-XVII. Madrid, Distinta Tinta, 2018, p. 17. 
importantes avances metodológicos y teóricos en el ámbito de la historia de las mujeres. Como consecuencia a estos impulsos, actualmente se encuentran presentes consideraciones, inquietudes y reflexiones sobre las mujeres y las relaciones de género en cualquier aspecto de la vida cotidiana y del espacio académico. Tradicionalmente la historiografía había justificado las dificultades para desarrollar investigaciones sobre la denominada «cuestión de la mujer» por la escasez o ausencia de fuentes. No obstante, actualmente estos impedimentos han sido superados «por la aplicación de unas herramientas analíticas apropiadas, las relaciones de género, y de una metodología acorde a las características del colectivo humano a estudiar». ${ }^{4}$

En este sentido, abordar el pasado de las mujeres en su cotidianeidad conlleva metodológicamente a un complejo análisis de las relaciones sociales en una determinada comunidad y al estudio de los distintos elementos que la conforman. Ahonda Baena Zapatero a este respecto en su estudio sobre la identidad de la mujer criolla. Deduce que la «invisibilidad» en las fuentes es debido al modelo patriarcal de la sociedad hispana de la época, la cual entendía la existencia de una división de funciones entre los hombres y las mujeres. Mientras que a los primeros les competía las actividades en la esfera pública, a las segundas les correspondía el cuidado del hogar y la reproducción. ${ }^{5}$ Consecuentemente, prosigue el autor, los historiadores contemporáneos interpretaron a estas mujeres como sujetos pasivos y ajenos de los grandes procesos sociopolíticos que debían ser estudiados ya que éstos habían sido gestados por hombres. ${ }^{6}$

Afirma Almorza Hidalgo que, a pesar de que el estudio de las movilidades dentro de los territorios coloniales castellanos a partir de las mujeres permite profundizar en los procesos migratorios, las circunstancias en el lugar de procedencia, las redes transfronterizas y las estrategias familiares, ${ }^{7}$ lo cierto es que han sido pocos los estudios sobre las emigrantes a la América castellana durante la Edad Moderna. La nesología, ${ }^{8}$ aplicada al ámbito espacial, y los

\footnotetext{
${ }^{4}$ MONZÓN PERDOMO, María Eugenia. "Vendederas en el comercio al por menor en la isla de Tenerife en el Antiguo Régimen. Entre el fraude y el control” In XVIII Coloquio de Historia Canario-Americana (2008). Las Palmas de Gran Canaria, Cabildo de Gran Canaria, 2010. p. 1884.

${ }^{5}$ BAENA ZAPATERO, op. cit., p. 137.

${ }^{6}$ Ibidem, p. 17.

${ }^{7}$ ALMORZA HIDALGO, Amelia. «No se hace pueblo sin ellas». Mujeres españolas en el virreinato de Perú: Emigración y movilidad social (Siglos XVI-XVII). Madrid, Consejo Superior de Investigaciones Científicas, 2018, p. 24.

${ }^{8}$ SANTANA PÉREZ, Juan Manuel. "Islas atlánticas en el comercio entre América y África en el Antiguo Régimen” In Cuadernos americanos. 2012, 142, p. 114.
} 
estudios atlánticos, desde una perspectiva cisatlántica, ${ }^{9}$ son los dos marcos teóricos que cobijan esta investigación sobre las mujeres portuguesas en Canarias. En este sentido, prosigue Almorza Hidalgo enfatizando que la Historia Atlántica ha demostrado su validez en el análisis de género. ${ }^{10}$ Aunque es una línea de trabajo poco desarrollada, la referida historiadora destaca los trabajos de Verena Stolke (2004), Bianca Premo (2010), Karen Graubart (2011) y Susan D. Amussen y Allyson M. Poska (2012) por el empleo de la categoría de género para realizar un análisis transimperial y la reivindicación de éstos de la centralidad del género en la organización y la articulación de los imperios atlánticos: «El género no solo proporciona la base para un análisis transimperial del mundo atlántico, sino que también nos permite reorientar nuestra perspectiva académica en el Atlántico». ${ }^{11}$ Además, para entender estas dinámicas atlánticas desde la categoría de género, como apunta Judith $\mathrm{P}$. Zinsser, es necesario repensar términos clásicos como «economía» o «redes de parentesco». ${ }^{12}$ En esta primera era de la globalización, por ejemplo, las dotes o los poderes otorgados a mujeres, como veremos más adelante, fueron una fuente importante de financiación para empresas familiares transatlánticas.

En el ámbito americano, la historiografía ha complejizado la visión sobre las mujeres indígenas, mestizas o negras. ${ }^{13}$ Sin embargo, son pocos los trabajos que, como el de Sullón Barreto, ${ }^{14}$ han tratado específicamente el proceso migratorio y la integración de forasteras. Sostiene Almorza Hidalgo que, «los estudios sobre los procesos de emigración trasatlántica han reconocido la importancia de la presencia femenina, si bien son escasos los análisis específicos sobre género y migraciones de larga distancia» ya que estos estudios se han centrado, ${ }^{15}$ como también ha defendido Baena Zapatero, en la movilidad masculina. ${ }^{16}$ Por tanto, concluye la citada historiadora, debemos incluir la categoría de género al análisis de estas movilidades familiares transatlánticas $\mathrm{y}$, para el caso de nuestro objeto de estudio, a las migraciones transnacionales.

\footnotetext{
${ }^{9}$ ARMITAGE, David. “Tres conceptos de historia atlántica” In Revista de Occidente. 2004, 281, p. 20.

${ }^{10}$ ALMORZA HIDALGO, op. cit., p. 31.

${ }^{11}$ AMUSSEN, Susan D. y POSKA, Allyson M. "Restoring Miranda: Gender and the Limits of European Patriarchy in the Early Modern Atlantic World" In Journal of Global History. 2012, 7, p. 342.

${ }^{12}$ ZINSSER, Judith P. “Women's History, World History, and the Construction of New Narratives” In Journal of Women's History. 2000, 12, p. 203.

${ }^{13}$ ALMORZA HIDALGO, op. cit., p. 31.

${ }^{14}$ SULLÓN BARRETO, Gleydi. “La presencia femenina entre los inmigrantes portugueses en Lima en el siglo XVII" In Revista complutense de historia de América. 2016, 42, pp. 267-292.

${ }^{15}$ ALMORZA HIDALGO, op. cit., p. 23.

${ }^{16}$ BAENA ZAPATERO, op. cit., p. 18.
} 
En este sentido, los estudios se han centrado en la concretización de las identidades, tanto la española o criolla en la América española como la portuguesa en el ultramar luso. Señala Herzog que la distinción entre lo «español»y «portugués» es una construcción ideológica posterior a la Restauración. Para el ámbito continental afirma que «la unión permitió la aparición de ciertas prácticas que, de hecho, en ocasiones, permitían ignorar la raya, confundiendo y enlazando lo que en teoría eran territorios separados o, al contrario, distinguiendo y dividiendo lo que debería de ser unificado». ${ }^{17} \mathrm{~A}$ este respecto, Monteiro y Cardim apuntan que estudiar este horizonte hispánico a partir de la simple dualidad entre Portugal y España no es suficiente para explicar la complejidad del escenario que estamos abordando. ${ }^{18}$

Afirma Hespanha que «existem poucas fontes de origem não oficial que façam a crónica desde outro império na sombra», a lo que apostilla que «falam mais destas comunidades periféricas as fontes estrangeiras do que as fontes portuguesas». ${ }^{19}$ En este contexto de expansión y consolidación de la presencia ibérica en espacios fronterizos concluye que los «portugueses» constituyen una «tribu» más entre todas las naciones que se mencionan en estas crónicas. En consecuencia, defiende Hespanha que el componente "portugués» en estos espacios alejados de la metrópoli es un elemento identificador en la comunidad a la que arriban, pero como otros tantos posibles, en el ámbito de las geometrías de identidad variables y relacionales de las sociedades locales. ${ }^{20}$

Aunque las personas podían estar clasificadas en grupos de acuerdo con definiciones jurídicas, estos agrupamientos no las determinaban del todo, ya que el hecho que más influía en el ordenamiento social se relacionaba con factores de solidaridad y colaboración humana. ${ }^{21}$ La naturaleza y la extranjería no eran un estatus fijo, sino más bien una clasificación que reflejaba la situación que unas personas tenían en relación con el grupo social que les rodeaba, este reflejo no sólo cambiaba continuamente sino que era además plurivalente. ${ }^{22}$

${ }^{17}$ HERZOG, Tamar. "Una monarquía, dos territorios. La frontera entre españoles y portugueses: España y Portugal durante (y después) de la Unión” In España y Portugal en el mundo (1581-1668) Madrid, Ediciones Polifemo, 2014, p. 148.

${ }^{18}$ MONTEIRO, Nuno Gonçalo y CARDIM, Pedro. "A centralidade da periferia. Prata, contrabando, diplomacia e guerra na região platina (1680-1806)” In História, histórias. 2013, 1, p. 3.

${ }^{19}$ HESPANHA, António Manuel. Filhos da Terra: Identidades mestiças nos confins da Expansão portuguesa. Lisboa, Tinta da China, 2019, p. 34.

${ }^{20}$ Ibidem, p. 37.

${ }^{21}$ HERZOG, Tamar. "La vecindad entre condición formal y negociación continua. Reflexiones en torno de las categorías sociales y las redes personales" In Anuario IEHS. 2000, 15, p. 125.

${ }^{22}$ HERZOG, Tamar. "Naturales y extranjeros: sobre la construcción de categorías en el mundo hispánico" In Cuadernos de Historia Moderna. 2011, 10, p. 27. 


\section{Interpretación y reinterpretación de las fuentes primarias insulares para el estudio de las mujeres portuguesas en Canarias}

Estos impulsos historiográficos y metodológicos aplicados a las fuentes primarias como herramientas para estudiar el pasado de las mujeres sustentan los resultados que presentamos en este trabajo. Esta investigación parte del análisis sistemático de la documentación canaria conservada para la época. En primer lugar, hemos acudido a las crónicas e historias contemporáneas insulares para establecer un primer abordaje a nuestro objeto de estudio. ${ }^{23}$ Estos cronistas coetáneos a la agregación portuguesa prestan atención hacia la comunidad lusa asentada en las Islas y revelan que son un sujeto dinámico en la sociedad canaria. Estos intervienen en el desempeño de las actividades económicas y en la construcción de una identidad insular a partir de aportes foráneos. Las mujeres portuguesas formaron parte de esta comunidad exógena que se asienta y se integra en Canarias. En definitiva, fueron agentes activos en la conformación de la sociedad insular y los cronistas percibieron y trasmitieron tanto las singularidades que mantienen como las aportaciones a la sociedad insular por parte de estas mujeres. Reflejo de esta rutina social a la que se incorpora la emigrante lusa lo encontramos en este breve pasaje de finales del siglo XVI del cronista azoriano Gaspar Frutuoso:

«[Las mujeres de la isla de La Palma] son muy hermosas, blancas y discretas, corteses y bien educadas, algunas están casadas con portugueses, otras con castellanos [...] bordan bien, pero casi no saben hilar ni tejer, cosa que dejan para las portuguesas». ${ }^{24}$

Aunque la información es escaza, como veremos por el estudio de otras fuentes primarias, estos relatos refieren al proceso de integración y asimilación de estas mujeres, pero también a la aptitud de éstas para la transferencia de conocimientos y experiencias, así como del bagaje cultural y profesional transnacional, desde el mundo portugués al espacio insular. En definitiva, a diferencia de otras fuentes de carácter oficial, el cronista como testigo de esta cotidianeidad plasma y visibiliza en su relato a las mujeres portuguesas como un agente activo, dinamizador en la sociedad canaria y portador de saberes e imágenes culturales exógenos que se convertirán en cotidianos y que formarán parte de la identidad de una sociedad fronteriza en construcción.

${ }^{23}$ Nos referimos a Gaspar Frutuoso, fray Alonso de Espinosa, Leonardo Torriani, Abreu Galindo y Núñez de la Peña.

${ }^{24}$ FRUTUOSO, Gaspar. Las islas Canarias (de "Saudades da Terra"). La Laguna, Instituto de Estudios Canarios, 1964, p. 109. 
Estas cuestiones que surgen a partir de una visión multifocal de la concomitancia entre el cosmos canario, la agencia femenina portuguesa y el universo ibérico en el Atlántico las hemos tratado acudiendo a fuentes primarias. En este sentido, el segundo paso en esta investigación ha sido el abordaje y el estudio de la documentación de archivo desde la perspectiva de género. Metodológicamente, hemos analizado la documentación insular inédita y hemos sistematizado todas aquellas referencias a mujeres portuguesas en los registros documentales que otros historiadores habían venido publicando como apéndice documental en sus trabajos.

En cuanto a las fuentes primarias inéditas, esta documentación constituye una herramienta indispensable para desarrollar un estudio de las migraciones transfronterizas, las relaciones sociales -tanto insulares como atlánticas- y la consolidación de redes transnacionales. Por el tipo de información que nos aporta y la variedad de asuntos que se trata, la documentación notarial para la isla de Tenerife es uno de los pilares sobre los que se sustenta esta investigación. ${ }^{25}$ Con todas las limitaciones y reservas, estas escrituras muestran la cotidianidad del individuo, su relación con el medio y la interacción con otros miembros de la sociedad. Para esta investigación realizamos un análisis de la documentación rubricada entre 1575 y 1650 con el objetivo de visualizar la evolución temporal de las movilidades de las mujeres hacia la Tenerife y reconstruir las relaciones familiares transatlánticas en momentos coyunturales del periodo la consolidación del mundo atlántico. En cuanto a las fuentes documentales que ya habían sido estudiados, nos referimos a aquellas que se habían venido referenciando en un conjunto de publicaciones, principalmente a lo largo de la década de 1990, y cuya finalidad era estudiar a la sociedad canaria en época moderna a partir de la compilación de información proveniente de documentos coetáneos, principalmente de fuentes inquisitoriales y notariales.

Por un lado, la particularidad principal de estos trabajos es que, si bien representan los primeros estudios de historia social aplicados al periodo del Antiguo Régimen en Canarias y se adentraron en el análisis de las minorías ${ }^{26}$ (extranjeras, religiosas, etc.) y de los grupos marginados y

\footnotetext{
${ }^{25}$ Escogimos Tenerife por dos cuestiones metodológicas. En primer lugar, el buen estado de conservación de la documentación para esta isla frente a otros territorios insulares del Archipiélago. En segundo lugar, por ser la isla con mayor proyección internacional durante esta época de la Edad Moderna.

${ }^{26}$ Citamos, como ejemplos, los trabajos de Anaya Hernández (1990) sobre minorías en Canarias, de Brito González (1993) sobre la presencia extranjera en Tenerife durante el Antiguo Régimen y de Fajardo Spínola (1998) sobre los protestantes extranjeros en Canarias.
} 
desprotegidos ${ }^{27}$ (esclavos, indigentes, etc.), las mujeres no fueron consideradas directamente como un sujeto de estudio. Éstas, las mujeres en Canarias y, en particular las foráneas, no fueron protagonistas ni de líneas de investigación ni de estudios específicos. Únicamente, en estas publicaciones científicas de finales del siglo XX, fueron integradas como parte de sus estudios en tanto que sus acciones complementaban sus objetos de investigación.

Por otro lado, también hay que tener en cuenta que, si bien estos investigadores pretendieron estudiar la complejidad de una sociedad de frontera como la canaria, la documentación que emplearon fue utilizada para resolver problemáticas históricas cuyos agentes eran principalmente masculinos (gestación de instituciones, actividad mercantil, etc.). Por tanto, sus trabajos se sustentaron, sin apenas cuestionárselo, en un relato exclusivamente construido por hombres.

A pesar de esta dificultad, consideramos que estos documentos contienen información suficiente para visibilizar la huella de las mujeres portuguesas en Canarias. En este sentido, nuestro objetivo es analizar nuevamente estas fuentes ya empleadas, pero en esta ocasión, con la finalidad de construir el discurso inclusivo en el que se incorpore a estas mujeres como agentes activos en la sociedad. Como refiere Baena Zapatero, no basta con «añadir» a las mujeres al relato preexistente. Se trata de construir nuevas narrativas y enfoques sobre antiguas preguntas. Cabe aquí, entonces, repensar conceptos en el ámbito insular y comparar procesos con otros espacios de la Monarquía Hispánica.

\section{La impronta de la mujer portuguesa en Canarias a través de la historiografía}

La aportación portuguesa a la población canaria, especialmente tras la integración de las Islas al reino de Castilla, ha sido un tema extensamente debatido por la historiografía. Rosa Olivera, a finales de la década de los 60 , expresaba por primera vez la relevancia cuantitativa del contingente portugués en el repoblamiento de determinadas zonas de la isla de Tenerife, concretamente en las comarcas noroccidentales de Icod y Daute. ${ }^{28}$ A partir

\footnotetext{
${ }^{27}$ Podemos referir los trabajos de Lobo Cabrera (1982) sobre la esclavitud en las Islas y el de Santana Pérez sobre la marginalidad en Canarias (2009).

${ }^{28}$ ROSA OLIVERA, Leopoldo de la. "El repoblamiento de los reinos de Icod y Daute" In Anuario del Instituto de Estudios Canarios. 1968-1970, 14-15, p. 39.
} 
de los registros de escribanos que consulta, ${ }^{29}$ concluye que el número de portugueses no es inferior en estas partes al 80\% del total de la población.

Una década más tarde se llevaron a cabo diversos estudios que dieron lugar a posturas diferentes y hasta opuestas en lo concerniente a la evolución del peso relativo de la comunidad portuguesa en comparación con otros grupos de inmigrantes establecidos en Canarias. Por un lado, FernándezArmesto, partiendo de una pesquisa con base en la documentación notarial de las islas de Tenerife y La Palma, ${ }^{30}$ y Aznar Vallejo, analizando las datas de repartimiento ${ }^{31}$ y las cartas de testamento, ${ }^{32}$ acentuaron la importancia numérica de los repobladores de origen lusitano, incluso por encima de los propios castellanos. Por el contrario, Charles Verlinden desconfiaba de estos datos estadísticos y defendía una integración mucho más matizada en la nueva sociedad isleña. ${ }^{33}$ Para este historiador, aunque el propósito inicial de estos primeros colonos era poblar el territorio, no todos permanecieron. En definitiva, plantea este investigador una presencia transitoria en el Archipiélago de estos portugueses, generalmente cristianos nuevos, de camino a otras partes de América como Caracas o Potosí.

Sobre la presencia de mujeres portuguesas en Canarias desde la agregación de la Isla a la corona de Castilla señala Bello León, en su investigación sobre los extranjeros en los repartimientos canarios, la relevancia de las familias portuguesas que emigraron a la isla de Tenerife para la consolidación de los asentamientos europeos. ${ }^{34}$ A este respecto, toma como objeto de

\footnotetext{
${ }^{29}$ No señala qué escrituras consulta, simplemente se limita a decir que abarcan estos registros desde 1513 hasta finales del siglo XVI.

${ }^{30}$ FERNÁNDEZ-ARMESTO, Felipe. Las Islas Canarias después de la conquista. La creación de una sociedad colonial a principios del siglo XVI. Las Palmas de Gran Canaria, Cabildo de Gran Canaria, 1982, p. 45.

${ }^{31}$ «De los 1.017 titulares de datas originales de la isla de Tenerife no llegan a 150 aquellos cuyo origen se distingue expresamente y de ellos 68 son canarios y 48 portugueses, cuya distinción resulta significativa, mientras que sólo tres son andaluces, número inferior al de vizcaínos e igual al de franceses». AZNAR VALLEJO, Eduardo. La integración de las Islas Canarias en la Corona de Castilla (1478-1526). Aspectos administrativos, sociales y económicos. Santa Cruz de Tenerife, Secretariado de Publicaciones de la Universidad de La Laguna, 1983, p. 194.

${ }^{32}$ «Más información ofrece una fuente indirecta, pero de gran fiabilidad, como es la respuesta por las cartas de testamento. De los 135 ejemplos examinados, 80 indican la procedencia del testador [...] El reparto de los 80 ejemplos conocidos es como sigue: 32 portugueses de diverso origen, 19 guanches, 7 castellanos, 7 extremeños, 6 andaluces, 2 canarios, 2 genoveses, 2 gallegos, 1 majorero, 1 genovés y 1 prieto». Ibidem, p. 194. ${ }^{33}$ VERLINDEN, Charles. "Castellanos, portugueses, italianos y otros pobladores de Canarias a raíz de la conquista. Una cuestión de proporciones" In VI Coloquio de Historia Canario-Americana (1984). Las Palmas de Gran Canaria, Cabildo de Gran Canaria, 1987, p. 14.

${ }^{34}$ BELLO LEÓN, Juan Manuel. "La participación de los extranjeros en los repartimientos canarios. Introducción a su estudio" In El Museo Canario. 1998, 53, p. 202.
} 
análisis a los Machado, familia lusitana natural de Guimarães, y «fundadora» del núcleo poblacional de Tacoronte. Indica este historiador que «parece que fue Lope Machado quien acordó su participación con Alonso de Lugo en la conquista de Tenerife, haciéndose acompañar a la misma por sus hijos Sebastián y Pedro ${ }^{35}$ El referido Sebastián estaba casado con la portuguesa Isabel González de Figueroa y que éstos, asevera el investigador, habrían sido los verdaderos impulsores del poblamiento de esta comarca y los fundadores de la ermita de Santa Catalina. ${ }^{36}$

Señala Almorza Hidalgo que «la emigración femenina hacia América constituye un caso excepcional dentro de las emigraciones transatlánticas y se trata del primer proceso en el que participaron las europeas». ${ }^{37}$ No obstante, hemos de recordar que desde inicios del siglo XV ya se había gestado un proceso migratorio desde Europa hacia el Atlántico con la expansión ibérica sobre la costa africana y, principalmente, sobre los archipiélagos atlánticos. Además, como pone de manifiesto el estudio de Bello León, en el caso de las islas que abordamos, esta emigración europea en los inicios de la expansión atlántica no solo sería de origen castellano.

Para las Islas Occidentales, en especial para Tenerife, han sido prácticamente inexistentes los trabajos que han tratado la representatividad del colectivo luso en relación con el conjunto de la población. El citado historiador Bello León (2002) elaboró un estudio sobre la comunidad portuguesa establecida en la ciudad de La Laguna durante las primeras décadas del siglo XVI. Sin embargo, apenas alude a la presencia de mujeres oriundas de Portugal. Los únicos datos proporcionados por la historiografía insular para el período de la Unión Ibérica han sido los extraídos del censo inquisitorial de $1626 .{ }^{38}$ En este padrón la mujer también tiene poca representatividad. Aparece visible únicamente cuando se refieren en el censo a la totalidad de los miembros de la unidad familiar. ${ }^{39}$ De las 95 personas censadas, nueve son mujeres. Éstas habrían emigrado a las Islas acompañando a sus maridos

\footnotetext{
${ }^{35}$ Ibidem, p. 201.

${ }^{36}$ Ibidem, p. 202.

${ }^{37}$ ALMORZA HIDALGO, op. cit., p. 21.

${ }^{38}$ FAJARDO SPÍNOLA, Francisco. "Portugueses en Canarias en el siglo XVII. Una relación de 1626" In XV Coloquio de Historia Canario-Americana (2002). Las Palmas de Gran Canaria, Cabildo de Gran Canaria, 2004, pp. 310-320.

${ }^{39}$ En este sentido, para el caso que nos ocupa, consultamos también las 33 «Informaciones de Solterías y Viudedades» disponibles en el Archivo Histórico Diocesano de San Cristóbal de La Laguna entre 1612 -fecha del primero que se conserva-y 1650. Igualmente, en los testimonios aportados en estos expedientes, únicamente se mencionan a portugueses varones y solteros, nunca a las mujeres.
} 
desde Portugal. No obstante, estas no son las únicas portuguesas a las que alude el censo. Indica Fajardo Spínola que dos tercios de los casados de los que tenemos datos contrajeron matrimonio en las Islas. ${ }^{40}$ En su mayoría con mujeres canarias, pero también con portuguesas o hijas de portugueses. En varios casos, con portuguesas que eran viudas de portugueses. Por tanto, continúa afirmando el autor, que existe «un proceso de integración social por vía matrimonial, pero también de un fenómeno de endogamia». Junto a estas nueve lusas que se establecen en la Isla, el censo menciona a otras nueve esposas de emigrantes portugueses que continuaban en Portugal. Este hecho, como señala el referido historiador, dio lugar a casos de bigamia. Los testimonios de este registro refieren a que dos de los casados en Canarias lo estaban también en Portugal. En este sentido, la bigamia fue uno de los principales delitos por el que se les acuse a las mujeres portuguesas ${ }^{41}$. La cercanía y los fuertes vínculos entre el Archipiélago y su entorno luso -insular y continental- habrían dificultado la ruptura definitiva de los lazos parentales. No obstante, como señala Baena Zapatero para el caso de las castellanas que emigraron a Indias, éstas «cargaban en su bagaje cultural los antecedentes de la bigamia, conocían perfectamente las restricciones que imponía el matrimonio y sabían cómo evitarlas». ${ }^{42}$

Cabe también resaltar para la obtención de datos sobre las mujeres portuguesas en Canarias el estudio llevado a cabo por Francisco Fajardo Spínola sobre las víctimas de la Inquisición en el Archipiélago. ${ }^{43}$ En la relación de individuos que expone en este trabajo se contabilizan un total de 90 portugueses entre 1580 y 1640, de los cuales 12 son mujeres. Todas ellas aparecen entre 1581 y 1629 , lo que demuestra la presencia constante y activa de este colectivo por lo menos hasta la ocupación holandesa del Brasil y la pérdida del principal mercado consumidor de los vinos canarios. Más aún, a partir de las referencias que utiliza, se observa el establecimiento de las mujeres portuguesas desde principios del siglo XVI y, por tanto, estos datos

\footnotetext{
${ }^{40}$ Ibidem, p. 212.

${ }^{41}$ Enfatiza Fajardo Spínola, en su estudio sobre las víctimas de la Inquisición en Canarias, la pequeña incidencia de los procesos por bigamia, más aún en una tierra de frontera e inmigración: 37 a lo largo del siglo XVI frente a los 485 por delito de proposiciones. No obstante, el mismo autor hace hincapié en el elevado número los procesados de origen portugueses, por encima incluso a los propios castellanos. Véase: FAJARDO SPÍNOLA, Francisco. "Las víctimas de la Inquisición canaria en el siglo XVI. Una aproximación cuantitativa", In XII Coloquio de Historia Canario-Americana (1996). Las Palmas de Gran Canaria, Cabildo de Gran Canaria, 1998, pp. 669-682.

${ }^{42}$ BAENA ZAPATERO, op. cit., p. 77.

${ }^{43}$ FAJARDO SPÍNOLA, Francisco. Las víctimas de la Inquisición en las Islas Canarias. La Laguna, Francisco Lemus Editor, 2005.
} 
confirmarían los tradicionales vínculos entre Portugal y Canarias. Así, si nos retrotraemos a una época anterior fueron procesadas por la Inquisición 23 lusas desde 1524 hasta 1579.

Estas cifras nos hablan de una emigración y de una presencia lusa en femenino en el Archipiélago. Más aún cuando comparamos la representatividad de las mujeres portuguesas con el de otras foráneas en Canarias. Sencillamente, durante todo el periodo cronológico de estudio, no hay ningún proceso o denuncia ante la Inquisición hacia otra foránea que no sea portuguesa.

Los datos extraídos nos indican que, en primer lugar, pese a la escasa información sobre mujeres forasteras en Canarias, entre éstas destacan cuantitativamente las portuguesas en las Islas frente a otras extranjeras europeas y, en segundo lugar, muestra el grado de integración de este colectivo dentro de la comunidad insular hasta casi asimilarse a un miembro más de la sociedad. Si bien son acusadas de judaísmo, este no es el principal delito por el que son procesadas. ${ }^{44}$ Son las proposiciones, seguidas de las supersticiones $\mathrm{y}$, en menor medida, por bigamia.

\section{La particular representación de la mujer insular portuguesa en la historiografía}

Durante la década de 1990 proliferaron los estudios sobre forasteros en el Archipiélago. Entre éstos tuvo especial relevancia los trabajos sobre el contingente migratorio desde Azores y Madeira a Canarias. Estas investigaciones se circunscribieron principalmente a las Islas Orientales (Gran Canaria, Lanzarote y Fuerteventura). Los trabajos de Lobo Cabrera y Torres Santana configuraron en las Islas una línea de investigación encaminada a reconstruir la composición de la sociedad insular empleando como método analítico la estadística poblacional y la cuantificación de registros. En este sentido, aunque en estas publicaciones las mujeres no fueron el objeto primordial de su investigación, la metodología empleada por estos dos historiadores nos ha permitido recuperar la agencia de las mujeres portuguesas en las fuentes primarias que emplearon.

A partir del análisis de las partidas matrimoniales de Gran Canaria entre 1585 y 1640 -repartidas entre los lugares de Las Palmas, Telde y La Vegaseñala Lobo Cabera que, en el 6,3\% de las nupcias, ${ }^{45}$ uno de los contrayentes

\footnotetext{
${ }^{44}$ De las 12 portuguesas procesadas por la Inquisición entre 1580 y 1640 solo dos son acusadas de judaísmo y seis entre las 23 denunciadas entre 1524 y 1579.

${ }^{45}$ Esta cifra corresponde a 175 matrimonios portugueses de un total en la Isla de 2746.
} 
era de origen portugués. Además, destaca en sus datos la presencia de los procedentes de las islas Azores, representando éstos el 26,3\% de la totalidad de portugueses. ${ }^{46}$

Hemos de subrayar la relevancia de los estos datos cuantitativos que aporta Lobo Cabrera para nuestro estudio. Por un lado, en el epígrafe que designa como «Sexo», refiere a que, para el conjunto de emigrantes azorianos que localiza en las fuentes primarias, la representación de las mujeres es mínima. Éstas supondrían escasamente el 8,7\% del contingente. Por otro, también destaca este historiador que en su mayoría estas mujeres se casaron con coterráneos suyos. Concretamente, menciona tres casos localizados de nupcias entre naturales de Terceira. En dos de estos acuerdos, ambos contrayentes son solteros, mientras que el otro lo conforma una pareja de viudos oriundos de aquella isla. Además, en un solo caso, cuyos progenitores son oriundos de Santa María, la mujer se casó con un vecino y natural de otra de las islas Canarias, en concreto Lanzarote. ${ }^{47}$

Por su parte, Torres Santana, en el análisis que realizó de las escrituras notariales de Lanzarote entre 1600 y 1640, localizó un total de 137 madeirenses y 28 azorianos presentes en dicha isla canaria. Esta cifra representaría, respectivamente, al 9,2\% y al 2,0\% de la población insular. Esta proporción habría alcanzado entre un $10 \%$ y un $15 \%$ si incluimos a los restantes lusos identificados en dicha documentación. ${ }^{48} \mathrm{Al}$ igual que en el estudio realizado por Lobo Cabrera, en las publicaciones de la historiadora Torres Santana las mujeres continúan siendo un apéndice en la investigación, limitándose a integrarlas en los datos cuantitativos a efectos demográficos. Por un lado, en su investigación sobre madeirenses en Lanzarote, resalta que las mujeres se presentan junto con sus maridos y que en «una sola ocasión aparece una mujer, Beatriz Rivera, sin ningún acompañante masculino». ${ }^{49}$ Para la referida investigadora, el hecho de que la emigración madeirense a Lanzarote fuese mayoritariamente masculina es indicativo de que los hombres tendrían

\footnotetext{
${ }^{46}$ LOBO CABRERA, Manuel. "Inmigrantes azoreanos en Gran Canaria durante el período filipino" In Boletim do Instituto Histórico da Ilha Terceira. 1987, 45, p. 1212.

${ }^{47}$ Ibidem, p. 1214.

${ }^{48}$ La investigadora cifra una población para Lanzarote que rondaría las 1500 personas hacia 1640 . TORRES SANTANA, Elisa. “Azoreanos en Lanzarote, 1600-1640" In História das Ilhas Atlânticas. (Arte, Comércio, Demografia, Literatura) Funchal, Centro de Estudos de História do Atlântico, 1997, p. 292.

${ }^{49}$ TORRES SANTANA, Elisa. “Lanzarote y Madeira durante la Unión Ibérica a través de la documentación notarial” In III Colóquio Internacional de História da Madeira, Funchal. Centro de Estudos de História do Atlântico, 1993, p. 652.
} 
facilidad para moverse entre islas mientras que las mujeres y sus familias podían seguir residiendo en su isla portuguesa.

De igual manera que en los otros estudios referidos, el número de mujeres portuguesas que encuentra en la documentación es exiguo. La autora localiza a seis mujeres que, como hemos indicado anteriormente, todas salvo una aparecen acompañando a sus maridos. Éstas representan el 4,4\% de la comunidad madeirense localizada en las fuentes estudiadas. ${ }^{50} \mathrm{Si}$ comparamos estas cifras con el conjunto de la población estimada en la isla de Lanzarote para la época, las mujeres procedentes de Madeira en Lanzarote no llegarían a representar al 0,5\% del contingente poblacional insular..$^{51}$

En su estudio sobre la emigración azoriana a la isla de Lanzarote entre 1620 y 1640 , esta historiadora localizó a 28 individuos oriundos del archipiélago portugués. De éstos, únicamente tres son mujeres. Es decir, un 16,6\% del total de estos emigrantes insulares y, por tanto, no superaría en ningún caso el 0,2\% en el conjunto de la población de Lanzarote. Si bien los datos cuantitativos no son excesivamente diferentes en comparación con el colectivo madeirense, sí es cierto que en este caso la proporción es significativamente mayor al triplicarse el porcentaje.

Aborda también esta investigadora la condición jurídica de estas azorianas que llegan a la isla de Lanzarote. Identifica a una vecina, una residente y una estante. Aunque cuantitativamente sigue siendo pobre, los testimonios que aporta nos revelan pautas y percepciones sobre los procesos de integración. Expone como ejemplo la experiencia vital de Ana de Acosta, quien había contraído matrimonio en Azores y que durante su estancia en Lanzarote enviudó y se volvió a casar. ${ }^{52}$ En la escritura que otorga declara que lleva más de diez años casada con su segundo marido por lo que, junto con los años del primer matrimonio, se puede apreciar que llevaba asentada en la Isla tiempo suficiente como para ser parte de la comunidad. Sin embargo, esta mujer se identifica en el documento como estante. Por tanto, debemos preguntarnos por qué no se consideró vecina y qué circunstancias impidieron la consolidación de su arraigo.

Junto con los registros notariales, los documentos inquisitoriales constituyen la principal fuente de información para rastrear la huella de

\footnotetext{
${ }^{50}$ De un total de 137 madeirenses. Ibidem, p. 650.

${ }^{51}$ Como señalamos anteriormente, Torres Santana indica que la población en Lanzarote «por esas fechas» sería de 1500 habitantes.
}

${ }^{52}$ TORRES SANTANA, Elisa. “Azoreanos en Lanzarote...”, p. 293. 
las mujeres portuguesas en Canarias. A este respecto, el trabajo de Fajardo Spínola y Anaya Hernández (1990) sobre las relaciones entre los archipiélagos atlánticos a partir de esta documentación visibiliza a otras mujeres emigrantes establecidas en el archipiélago canario, en este caso las procedentes de las islas portuguesas. Más del $80 \%$ de las 26 denuncias o procesos que estudian en este trabajo contra naturales de Madeira y Azores para los siglos XVI y XVII son pleitos contra las mujeres. ${ }^{53}$ Dos tercios se situarían en el periodo de la agregación portuguesa a la Monarquía Hispánica y más de la mitad fueron acusadas y apresadas en Lanzarote..$^{54}$ Por un lado, de estos datos inferimos que la agencia de las mujeres lusas en la sociedad canaria fue igual de relevante y activa que la masculina, incluso mayor en algunos ámbitos. Por otro lado, observamos la importancia como nexo de la isla de Lanzarote y el entorno portugués en el periodo álgido de las relaciones luso-canarias. Asimismo, estas mujeres ostentaron un papel fundamental en la consolidación de los vínculos sociales trasatlánticos desde un territorio de frontera.

Hemos indicado que ninguno de estos trabajos tomó a las mujeres como objeto de estudio. Aparecen como un elemento cuantitativo más y su historia órbita alrededor de la de los hombres. En el mejor de los casos, estas mujeres están representadas en las tablas y anexos de estos trabajos. Los datos no se pueden comparar a la huella dejada por los hombres. Sería infructuoso confrontar el número de escrituras notariales de unos y de otras porque porcentualmente las mujeres y, especialmente las foráneas, apenas otorgaron escrituras. Sin embargo, las referencias que dejaron han permitido recuperar su agencia en la sociedad canaria. Éstas hablan de una emigración y de una presencia lusa en femenino en el Archipiélago.

\section{Antiguos estudios, fuentes inéditas y nuevos resultados: La huella de la mujer portuguesa en la sociedad canaria}

Estas aproximaciones estadísticas en relación con la presencia extranjera -y en especial la portuguesa- en las Canarias Orientales fueron objeto de un estudio más amplio por parte de Brito González (2002). Muestra su investigación, elaborada a partir del estudio de la documentación notarial insular, que los individuos de origen portugués en la isla de Gran

\footnotetext{
${ }^{53}$ Concretamente, 23 mujeres procedían Madeira y 3 de Azores.

${ }^{54}$ ANAYA HERNÁNDEZ, Luis Alberto y FAJARDO SPÍNOLA, Francisco (1990), "Relaciones de los archipiélagos de Azores y Madera con Canarias, según fuentes inquisitoriales (siglos XVI y XVII)" In I Colóquio Internacional de História da Madeira. Funchal, Centro de Estudos de História do Atlântico, 1990, p. 851.
} 
Canaria durante el siglo XVII constituyen casi el 36\% de los extranjeros. Esta preponderancia fue aún más elevada en Lanzarote, donde los lusitanos suponen el $70 \%{ }^{55}$ Asimismo, indica que la mayoría se circunscribe al período de la agregación portuguesa a la Monarquía Hispánica, entre 1618 y 1640. Estos datos vislumbran, en el territorio canario, la relevancia de esta comunidad en la conformación de la sociedad insular. Aún más, este análisis en el ámbito local acompaña a otros estudios sobre el espacio americano que demuestran la agencia de determinados grupos exógenos en el proceso de construcción de identidades en espacios de frontera. ${ }^{56}$

Aunque Brito González no realiza un análisis de la presencia de mujeres foráneas en las Islas, extraemos datos cuantitativos sobre las mujeres portuguesas en las Canarias Orientales de la relación nominal de «extranjeros» que aporta en el anexo que complementa su publicación. En primer lugar, advertimos la relevancia cuantitativa de las mujeres portuguesas en las Islas, frente a otras extranjeras en Canarias y también en comparación con otras lusas asentadas en la América española. Por un lado, según sus fuentes consultadas, las mujeres foráneas no ibéricas se asentaron en la isla de Gran Canaria y procedieron de Inglaterra (2), Francia (1), Génova (1), Flandes (1) y Ámsterdam (1). Estos datos hay que tomarlos con cautela ya que el estudio que realiza el historiador Brito González aborda todo el siglo XVII y sabemos que a partir de la segunda mitad de la centuria disminuye el número de portugueses y se consolida la presencia de otras comunidades forasteras en las Islas, principalmente del norte de Europa. Aun así, con todas las reservas, si contraponemos estas cifras de forasteras no ibéricas con las aportadas para el contingente de mujeres portuguesas que llegan a las Canarias Orientales atestiguamos el predominio de estas últimas. Si en nuestro estudio para la isla de Tenerife ubicamos a diez mujeres lusas en esta isla, el citado historiador aporta cifras similares.$^{57}$ Localiza entre los registros notariales que consulta para la isla de Gran Canaria a 12 foráneas, de las cuales la mitad son portuguesas. En la isla de Lanzarote ubica a 15 forasteras, todas portuguesas. Por su parte, en Fuerteventura no le consta la presencia de mujeres de otros reinos entre los 33 individuos extranjeros que rescata de las fuentes.

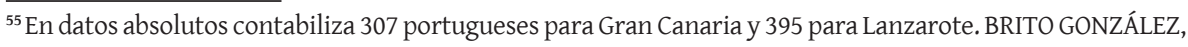
Alexis D. Los extranjeros en las Canarias Orientales en el siglo XVII. Las Palmas de Gran Canaria, Cabildo de Gran Canaria, 2002, p. 46.

${ }^{56}$ Sobre la presencia e integración de la comunidad portuguesa en la América española, véase los trabajos de Drumond Braga (1995), Ceballos (2008) y Serrano Mangas (1997; 2001).

${ }^{57}$ A partir de la documentación notarial consultada para el período 1575-1650 hemos localizado un total de 593 individuos de procedencia portuguesa que actuaron ante las escribanías de Tenerife.
} 
Por otro lado, la historiadora Sullón Barreto, a partir del abordaje que realiza de la documentación notarial peruana, halla en Lima entre 1600 y 1680 a ocho mujeres portuguesas. ${ }^{58}$ Por su parte, Brito González, únicamente para dos de las ocho islas que componen el Archipiélago localiza para todo el siglo XVII a 21 mujeres portuguesas. En comparación, la isla de Gran Canaria casi iguala las cifras reportadas para la Capital de los Reyes mientras que Lanzarote casi duplica el número de mujeres portuguesas. Es decir, la población de mujeres lusas en estas dos únicas islas supera a Lima en 262,5\%. Esta proporción sería aún mayor si incluyésemos a las mujeres portuguesas que hemos localizado en la isla de Tenerife en la documentación notarial trabajada. En consecuencia, aunque nos encontramos ante valores cuantitativos bajos, inferimos que la proporción de mujeres lusas en espacios insulares geográficamente limitados es alta en comparación con otros territorios coloniales castellanos en América.

En segundo lugar, a pesar de todas las consideraciones que hemos advertido sobre el acceso y participación en las escrituras notariales de las mujeres, confirmamos que la emigración portuguesa hacia el Atlántico castellano es mayoritariamente masculina. En Gran Canaria las mujeres representan únicamente el 1,95\% de la comunidad portuguesa en la isla. Esta cifra muy similar a la que hemos obtenido en nuestro estudio sobre la isla Tenerife, con un 1,68\%. Mientras, esta representación es algo mayor en Lanzarote con un 3,79\%. Estos porcentajes son similares a los datos obtenidos para otros espacios atlánticos. Así, en Lima esta proporción alcanza el $4,85 \%$ para todo el siglo XVII. ${ }^{59}$ Finalmente, si cotejamos estos datos con los proporcionados por Almorza Hidalgo para la emigración transatlántica entre la Península Ibérica y América, observamos que la movilidad de las mujeres entre espacios sujetos a la misma soberanía era sustancialmente mayor. Indica la referida historiadora que estas mujeres llegaron a suponer en $1580 \mathrm{casi}$ el $30 \%$ de los emigrantes a Indias, aunque en la década de 1620 estas movilidades sufrieron un descenso radical..$^{60}$ En cualquier caso, se observa que esta la mayor parte de los desplazamientos hacia territorios castellano en el Atlántico fueron realizados, ya fuesen éstos naturales del reino o forasteros. Para Almorza Hidalgo, la emigración femenina tuvo un desarrollo propio condicionado por la movilidad del grupo familiar, las condiciones de salida y de acogida y la necesidad de garantizar la cobertura de seguridad. ${ }^{61}$

\footnotetext{
${ }^{58}$ SULLÓN BARRETO, Gleydi. "La presencia femenina...”, p. 269.

${ }^{59}$ Ibidem, p. 269.

${ }^{60}$ ALMORZA HIDALGO, op. cit., p. 58.

${ }^{61}$ Ibidem, pp. 91-93.
} 
En tercer lugar determinamos, por los datos que extraemos tanto del trabajo de Brito González como de nuestro estudio con fuentes inéditas, que el lugar de procedencia es mayoritariamente insular portugués. En el caso de Gran Canaria la mitad de estas lusas procedieron de las islas. En concreto, dos de Madeira y otra de Fayal. Respecto al resto de mujeres portuguesas en esta isla canaria, una es oriunda de Lisboa y las otras dos se identificaron genéricamente como «portuguesas» sin precisar su lugar origen. Mientras, para Lanzarote, Brito González localizó a nueve lusas oriundas de Madeira y otra de Porto Santo, además de dos azorianas. Las otras tres aparecen referidas con el término «portuguesas». En el caso de Tenerife, estas mujeres emigrantes vienen de lugares del Portugal continental, tanto del interior -como Portalegre- como de las regiones costeras de Beira Litoral y Minho. Al igual que para las otras dos islas canarias, también aparecen representados los espacios insulares portugueses -como la isla de Santa María en Azorescomo lugar de origen. ${ }^{62}$ Esta disparidad de lugares en los orígenes nos permite aseverar que Tenerife, más que en los casos estudiados para Gran Canaria o Lanzarote, mantuvo una proyección internacional mayor y, en consecuencia, propició la arribada de mujeres de diversas partes de Portugal en busca de un lugar más próspero y con mayores oportunidades.

En cuarto lugar, advertimos una correspondencia entre el lugar de establecimiento con la actividad desarrollada por la unidad familiar, incluidas las mujeres. Localizamos a todas las forasteras en Gran Canaria asentadas en la ciudad de Las Palmas de Gran Canaria, salvo una portuguesa que se establece en Guía. Los lugares de arraigo en la isla de Tenerife son algo más dispares, aunque los datos apuntan a una mayor presencia de mujeres en las zonas urbanas de la capital y su comarca -cuatro en La Laguna y una en Tacoronte-, del Valle de la Orotava y del puerto de Garachico. Resulta complejo visibilizar a través de las fuentes empleadas a mujeres en áreas rurales por la escasez de registros y por las dificultades para actuar de éstas ante escribanos, por lo que deducimos que la representación que aquí se muestra es el reflejo de un establecimiento en áreas urbanas en el que se desarrolla la actividad mercantil a nivel local e internacional. Estos datos no implican que el asentamiento de estas mujeres fuese mayoritariamente urbano, ya que sabemos que la principal actividad desempañada por portugueses es la labor campesina y ésta está ligada al mundo rural, sino que el número de escrituras y la tipología

\footnotetext{
${ }^{62}$ En un reciente trabajo publicado por el investigador González Marrero (2019) refiere a 13 mujeres azorianas que se habrían establecido en la población de La Orotava en Tenerife a lo largo del siglo XVI. Además, salvo una de ellas, todas habrían llegado a la isla antes de 1580.
} 
documental en las ciudades y puertos es mucho mayor debido a la diversidad y cantidad de actividades que en estos espacios se desarrollaron.

Las actividades comerciales, por lo menos las que mencionan la documentación, son mayoritariamente de hombres. No obstante, detectamos a través de estos datos otra particularidad de las mujeres portuguesas. Si bien podemos inferir que la llegada de extranjeras y la elección de su lugar de establecimiento están asociados a la actividad económica de su familia y no se les reconoce a éstas ningún oficio, nos encontramos con una singularidad entre las portuguesas. Estas fueron las únicas forasteras a las que se les reconoce una profesión, la de vendedoras. En el caso del estudio de Brito González, éste alude a cinco vendedoras oriundas de Portugal establecidas en Gran Canaria y Lanzarote a lo largo del siglo XVII. En la isla de Tenerife, tres de las siete avecindadas en la Isla tenían oficio y una de ellas se definía como «mujer libre». Estas mujeres se dedican al pequeño comercio, también como vendedoras ${ }^{63}$ o como tenderas de vino y aceite. ${ }^{64}$ Si bien el ejercicio de esta profesión por parte de las mujeres portuguesas es visible en la documentación notarial principalmente a partir de las escrituras de fianzas, otorgadas por hombres a estas mujeres como avalistas de su oficio, ${ }^{65}$ encontramos también en esta fuente documental referencias a que estas mujeres vendedoras forjaron un negocio junto a sus maridos ${ }^{66} \mathrm{e}$, incluso, llegaron a conformar una red mercantil internacional. Este es el caso de María Luis, vendedora portuguesa asentada en La Laguna, que en el año 1612 era deudora de 85 reales a un mercader portugués vecino de Tavira. ${ }^{67}$

Señala Baena Zapatero, para el ámbito novohispano, que las criollas «lejos de cumplir a rajatabla el modelo patriarcal establecido» aparecieron en el espacio público y que, por tanto, en contra del modelo historiográfico que se había apuntado, estas mujeres habrían participado activamente más allá del ámbito doméstico. Cierto es que, en el caso de Canarias, las castellanas ejercieron también algunos oficios públicos. No obstante, no

\footnotetext{
${ }^{63}$ Sobre el ordenamiento del oficio de vendedoras en Tenerife, véase el trabajo de Monzón Perdomo (2010) sobre las vendederas en el comercio al por menor en la isla de Tenerife en el Antiguo Régimen.

${ }^{64}$ Es el caso de Catalina González, portuguesa vecina de Garachico. A.H.P.S.C.T., Sección Histórica de Protocolos Notariales, legajo 2085, sin fol.

${ }^{65}$ Sirva como ejemplo la fianza otorgada por Diego Monsalve a la portuguesa María Hernández la preñada para que pudiese vender en su tienda. A.H.P.S.C.T., Sección Histórica de Protocolos Notariales, legajo 920, sin fol.

${ }^{66}$ En 1614 el alcalde de Tacoronte reconocía que se había enterrado a Elena González, portuguesa, y que tanto ella como su marido habían sido vendedores y describía un inventario de bienes dejado por la difunta por un valor de 2.130 reales. A.H.P.S.C.T., Sección Histórica de Protocolos Notariales, legajo 1532, f469. ${ }^{67}$ A.H.P.S.C.T., Sección Histórica de Protocolos Notariales, legajo 1532, fำ30.
} 
nos consta, por las mismas fuentes consultadas, el desempeño de oficios de mujeres no castellanas que no fueran lusas ni que ejercieran la actividad mercantil a escala internacional. Sin embargo, por el tipo de documentación empleada, estos datos deben ser puntualizados. Entendemos que este modelo de mujer emprendedora representó una minoría, pero es justamente esta excepcionalidad lo que hace que aparezcan reflejadas en tales escrituras.

Señala Santana Pérez, sobre las vendedoras en La Laguna a mediados del siglo XVII que

como siempre en estos casos, en algunas de ellas se recoge su mote o alguna característica física o de su origen. Así aparecen algunas con alias de la parga, la pintora, la vizcaína, harnenera, o la abadesa e igualmente algunas características como ser negra, portuguesa o natural de Canaria. ${ }^{68}$

Sin duda alguna, este oficio fue desempeñado por un número significativo de portuguesas y así la documentación de la época lo refleja. No obstante, ni todas las portuguesas fueron vendedoras ni todas las vendedoras tuvieron un origen luso. En la documentación consultada hemos localizado a mujeres lusas casadas con artesanos, agricultores y mercaderes que, muy probablemente, trabajarían o colaborarían con las labores de sus maridos.

Si comparamos estos datos con la Ciudad de los Reyes, las fuentes estudiadas por Sullón Barreto indican que «en su mayoría practicaron el comercio a pequeña escala [...] También sabemos que algunas alternaron este comercio con el préstamo de capital». ${ }^{69}$ Refiere además que

como mujeres autónomas -ya en la gestión de sus negocios o en la administración y disposición de sus bienes-, [...] se hallaban totalmente integradas en el medio, creaban vínculos interpersonales con los otros vecinos -naturales del reino y otros extranjeros-, y desarrollaban también cierto sentido de pertenencia. ${ }^{70}$

Si bien podemos inferir pautas similares en el archipiélago, en cuanto a la administración de bienes, la gestión comercial y la consolidación de vínculos transfronterizos, no observamos que, en el entorno limeño las

\footnotetext{
${ }^{68}$ SANTANA PÉREZ, Germán. “¿Capacidad o sumisión comercial?: Vendedoras canarias durante el siglo XVII" In Boletín Millares Carlo. 2002, 21, p. 44.

${ }^{69}$ SULLÓN BARRETO, Gleydi. Vasallos y extranjeros. Portugueses en la Lima virreinal, 1570-1680. Madrid, Universidad Complutense, 2014, p. 278.

${ }^{70}$ Ibidem, p. 275.
} 
mujeres portuguesas, además del desempeño de estas actividades económicas, tuvieran un oficio o empleo reconocido como en Canarias.

En cuarto lugar, detectamos diferencias en el proceso de arraigo e integración de las portuguesas según el lugar de asentamiento. La consolidación del establecimiento estuvo condicionada a la fortaleza de los vínculos que el lugar de acogida hubiese mantenido con el entorno portugués. En el caso de Gran Canaria, observamos que, de las cuatro que conocemos su estatus jurídico, todas fueron vecinas. En la isla de Tenerife, la mayor parte de las mujeres -siete- se definen como vecinas frente a tres estantes. Sin embargo, en la isla de Lanzarote, apenas fueron localizadas cuatro vecinas lusas. En esta última isla, diez de estas mujeres se declararon residentes o estantes en Lanzarote. Este contraste entre espacios tan cercanos debe entenderse, como señalaba Torres Santana, por la tradicional relación entre Lanzarote y los territorios portugueses, principalmente los insulares. A este respecto, la isla majorera actuaría de trampolín hacia otros territorios ibéricos mientras que Gran Canaria y Tenerife serían lugares más proclives para un establecimiento a largo plazo y el desarrollo de actividades que consolidasen el arraigo.

En quinto y último lugar, se aprecia que las mujeres forasteras que emigraron a las Islas realizaron este desplazamiento y consolidaron su arraigo de acuerdo con los intereses de la estructura familiar. En este sentido, a partir de los datos que proporciona Brito González, concluimos que, salvo una excepción, ${ }^{71}$ todas las foráneas que llegaron tanto a Gran Canaria como a Lanzarote y que indicaron su situación civil refieren a que están casadas o son viudas. En el caso de las portuguesas, ocho mencionan que están casadas y una refiere que es viuda. Desconocemos, por los datos que proporciona el autor, si estas mujeres en estas dos islas contrajeron matrimonio con coterráneos o con castellanos y si las uniones se concertaron en Portugal o Canarias, que estas mujeres fueron el nexo entre la sociedad que les acoge y la comunidad lusa de procedencia. A este respecto, de las mujeres que arraigaron en Tenerife y conocemos su estatuto jurídico, todas se encuentran casadas. Por el contrario, de las que no se encuentran avecindadas, es significativo que de la única de la que disponemos información estuviese soltera. ${ }^{72}$ Fue el caso de Ana Antonia, un testimonio singular. Su testamento nos informa que su llegada a la Isla se habría realizado recientemente, cinco años antes de que otorgara la escritura

\footnotetext{
${ }^{71}$ Una mujer soltera procedente de Flandes.

${ }^{72}$ Difieren estos datos con los proporcionados por Almorza Hidalgo para la emigración castellana a América. Esta historiadora señala que, si bien hubo un descenso a finales del siglo XVI, el 60\% de las emigrantes eran solteras y el $40 \%$ restante eran casadas o viudas. ALMORZA HIDALGO, op. cit., p. 71.
} 
en 1624. Ella misma menciona que no está casada ni ha tomado estado, por lo que no deja herederos ni ascendientes. No obstante, pertenece a una unidad familiar establecida en el territorio insular. Al menos mantiene trato con primo hermano suyo, un clérigo presbítero, con el que tiene ciertas deudas y al que deja como albacea. ${ }^{73}$

En particular para la isla de Tenerife, hemos localizado el testamento de tres mujeres oriundas de Portugal. Dos de ellas solteras y la otra casada, cuyo marido se encontraba en Cabo Verde. ${ }^{74}$ Precisamente esta última escritura hace referencia a la última voluntad de Blanca Rodríguez, oriunda de la región de Oporto y residente en Garachico, y nos permite observar el nivel de integración de estas forasteras en la sociedad insular. Además de indicar que un zapatero y un molinero de la zona donde se asienta le eran deudores de cierta cantidad de reales, mandó una dobla de sus bienes a la cofradía de la Misericordia, de la que dijo que era cofrade, y pidió que su cuerpo fuese sepultado en el monasterio del señor San Francisco de Garachico. ${ }^{75}$ Estas pautas expresadas en su testamento indican que esta mujer formó parte activa de la comunidad de acogida, tanto en el ámbito económico como social.

Los poderes notariales son otra fuente de información para analizar la conciencia de arraigo y naturalización de una buena parte de los portugueses. Hemos localizado un tal de 47 de este tipo de escrituras en los que reclamaban bienes y herencias de familiares en sus regiones de procedencia. Las mujeres portuguesas asentadas en la Isla confirieron su poder a aquellas personas más o menos cercanas, siempre hombres, que estaban de paso por Canarias y se dirigían al lugar de origen del otorgante, haciendo de esta forma fluir la red familiar. También articularon las relaciones socioeconómicas ya que fueron éstas, a través de sus poderes, las que otorgaron la potestad para vender o arrendar los bienes obtenidos por herencia, incluso de interferir en las posibles disputas familiares.

Es revelador que, una decena de estas escrituras, las solicitantes son las mujeres. Este es el caso de Ana María, viuda de Gaspar Rodríguez y vecina de Icod. En 1603 otorgó un poder a Antonio de Castro, residente en Garachico de próximo viaje a Santiago de Cabo Verde, para que en aquella isla pudiese

\footnotetext{
${ }^{73}$ A.H.P.S.C.T., Sección Histórica de Protocolos Notariales, legajo 3411, f445.

${ }^{74}$ Las cifras son similares si comparamos los datos con los obtenidos por Almorza Hidalgo. Para la ciudad de Lima, el $5 \%$ de los testamentos otorgados durante el siglo XVII pertenecieron a mujeres. En el ámbito indiano, la mayoría de estas testadoras eran viudas o casadas, aunque en una proporción similar a las solteras. ALMORZA HIDALGO, op. cit., p. 269.
}

${ }^{75}$ A.H.P.S.C.T., Sección Histórica de Protocolos Notariales, legajo 2066, fo338. 
cobrar cualquier cantidad «de cueros, vinos, azúcar o esclavos que su marido haya dejado o le deban en Cabo Verde, donde falleció»». ${ }^{76}$

Entendemos que las mujeres fueron un nexo esencial entre la comunidad portuguesa establecida en diferentes espacios del Atlántico. Estas trasmisoras del patrimonio común fueron principalmente esposas o viudas del patriarca familiar, pero también las hijas y las hermanas fueron difusoras del legado de la estirpe. En este sentido, citamos como ejemplo el poder otorgado en 1614 por un madeirense estante en Tenerife a fray Fernando de San Gregorio de la orden de predicadores, y su prior del convento de Santa María de la Real de Candelaria, para que en su nombre y en el de sus hijas Beatriz Tejera y Juana Tejera, así como en el de su mujer difunta Clara Rodríguez, recibiese y cobrase de un pedazo de viña que él y sus hijas tenían en Madeira.

\section{Conclusiones}

Si difícil ha sido recuperar el rastro de la mujer, más aún lo ha sido el de la mujer forastera. Su doble condición entorpeció enormemente que tuviera posibilidad de iniciativa, por lo menos individualmente. Una parte importante de los emigrantes portugueses fueron estantes en las islas, lo que limitó sus acciones en la sociedad. Sencillamente, no formaban parte de ella. En este contexto, la mujer portuguesa dependía de un hombre, con el que había emigrado o se había casado ya en la isla, para integrarse en la nueva sociedad. Por tanto, debía combatir contra el recelo por ser extranjera y por ser mujer.

Las escrituras, aunque pocas, otorgadas por mujeres portuguesas en el ámbito insular indican que fueron indispensables en la organización familiar, el sostenimiento de vínculos transfronterizos entre parientes y en la consolidación de redes socioeconómicas atlánticas. Éstas fueron reconocidas socialmente como individuos con derechos, suficientes como para rubricar un acuerdo en su propio nombre y en el de sus herederos. Además, excepcionalmente fueron reconocidas como trabajadoras y con oficio, el de vendedoras. Estas circunstancias demuestran que, además de mujeres y portuguesas, fueron también insulares al formar parte activa de la sociedad.

Las mujeres fueron el nexo entre los distintos miembros de la familia, establecidos a uno y otro a lado de la frontera luso-castellana. Éstas administraron los bienes familiares y fueron a su vez difusoras del legado familiar y, en último término, de una identidad. Las mujeres lusas fueron el

${ }^{76}$ A.H.P.S.C.T., Sección Histórica de Protocolos Notariales, legajo 684, fo ${ }^{154 v . ~}$ 
eslabón entre el lugar de origen y el nuevo hogar, entre el forastero y el local, entre las viejas y las nuevas generaciones. En definitiva, las mujeres fueron las encargadas de mantener y trasmitir la memoria de la familiar en un territorio ajeno y distante del hogar primigenio.

A partir del análisis de fuentes primarias inéditas y de la revisión de registros documentales utilizados por otros autores para abordar otros objetos de estudio, hemos podido elaborar un estudio aproximativo sobre las características que definieron la identidad de las mujeres portuguesas en Canarias.

\section{Referencias}

ALMORZA HIDALGO, Amelia. «No se hace pueblo sin ellas». Mujeres españolas en el virreinato de Perú: Emigración y movilidad social (Siglos XVI-XVII). Madrid, Consejo Superior de Investigaciones Científicas, 2018.

ÁLVAREZ SANTOS, Javier Luis. "Identidad y pacto social: los portugueses en Canarias durante la Unión Ibérica" In Anuario de Historia Regional y de las Fronteras. 2019, 24, pp. 139-154.

AMUSSEN, Susan D. y POSKA, Allyson M. "Restoring Miranda: Gender and the Limits of European Patriarchy in the Early Modern Atlantic World" In Journal of Global History. 2012, 7, p. 342-363.

ANAYA HERNÁNDEZ, Luis Alberto y FAJARDO SPÍNOLA, Francisco (1990), "Relaciones de los archipiélagos de Azores y Madera con Canarias, según fuentes inquisitoriales (siglos XVI y XVII)" In I Colóquio Internacional de História da Madeira. Funchal, Centro de Estudos de História do Atlântico, 1990, p. 846-877.

ARMITAGE, David. "Tres conceptos de historia atlántica" In Revista de Occidente. 2004, 281, p. 7-28.

AZNAR VALLEJO, Eduardo. La integración de las Islas Canarias en la Corona de Castilla (1478-1526). Aspectos administrativos, sociales y económicos. Santa Cruz de Tenerife, Secretariado de Publicaciones de la Universidad de La Laguna, 1983.

BAENA ZAPATERO, Alberto. Mujeres novohispanas e identidad criollas. S. XVI-XVII. Madrid, Distinta Tinta, 2018.

BELLO LEÓN, Juan Manuel. "La participación de los extranjeros en los repartimientos canarios. Introducción a su estudio” In El Museo Canario. 1998, 53, p. 189-213. 
FAJARDO SPÍNOLA, Francisco. "Las víctimas de la Inquisición canaria en el siglo XVI. Una aproximación cuantitativa", In XII Coloquio de Historia Canario-Americana (1996). Las Palmas de Gran Canaria, Cabildo de Gran Canaria, 1998, pp. 669-682.

FAJARDO SPÍNOLA, Francisco. "Portugueses en Canarias en el siglo XVII. Una relación de 1626" In XV Coloquio de Historia Canario-Americana (2002). Las Palmas de Gran Canaria, Cabildo de Gran Canaria, 2004, pp. 310-320.

FAJARDO SPÍNOLA, Francisco. Las víctimas de la Inquisición en las Islas Canarias. La Laguna, Francisco Lemus Editor, 2005.

FERNÁNDEZ-ARMESTO, Felipe. Las Islas Canarias después de la conquista. La creación de una sociedad colonial a principios del siglo XVI. Las Palmas de Gran Canaria, Cabildo de Gran Canaria, 1982.

FRUTUOSO, Gaspar. Las islas Canarias (de "Saudades da Terra"). La Laguna, Instituto de Estudios Canarios, 1964.

BRITO GONZÁLEZ, Alexis D. Los extranjeros en las Canarias Orientales en el siglo XVII. Las Palmas de Gran Canaria, Cabildo de Gran Canaria, 2002.

HERZOG, Tamar. "La vecindad entre condición formal y negociación continua. Reflexiones en torno de las categorías sociales y las redes personales" In Anuario IEHS. 2000, 15, p. 123-131.

HERZOG, Tamar. "Naturales y extranjeros: sobre la construcción de categorías en el mundo hispánico” In Cuadernos de Historia Moderna. 2011, 10, p. 21-31.

HERZOG, Tamar. "Una monarquía, dos territorios. La frontera entre españoles y portugueses: España y Portugal durante (y después) de la Unión" In España y Portugal en el mundo (1581-1668) Madrid, Ediciones Polifemo, 2014, p. 139-155.

HESPANHA, António Manuel. Filhos da Terra: Identidades mestiças nos confins da Expansão portuguesa. Lisboa, Tinta da China, 2019.

LOBO CABRERA, Manuel. "Inmigrantes azoreanos en Gran Canaria durante el período filipino" In Boletim do Instituto Histórico da Ilha Terceira. 1987, 45, p. 1207-1222.

MONTEIRO, Nuno Gonçalo y CARDIM, Pedro. “A centralidade da periferia. Prata, contrabando, diplomacia e guerra na região platina (1680-1806)” In História, histórias. 2013, 1, p. 3-22

MONZÓN PERDOMO, María Eugenia. "Vendederas en el comercio al por menor en la isla de Tenerife en el Antiguo Régimen. Entre el fraude y el control" In XVIII 
Coloquio de Historia Canario-Americana (2008). Las Palmas de Gran Canaria, Cabildo de Gran Canaria, 2010. p. 1388-1405.

ROSA OLIVERA, Leopoldo de la. "El repoblamiento de los reinos de Icod y Daute" In Anuario del Instituto de Estudios Canarios. 1968-1970, 14-15, p. 35-43.

SANTANA PÉREZ, Germán. “¿Capacidad o sumisión comercial?: Vendedoras canarias durante el siglo XVII” In Boletín Millares Carlo. 2002, 21, p. 41-49.

SANTANA PÉREZ, Juan Manuel. "Islas atlánticas en el comercio entre América y África en el Antiguo Régimen” In Cuadernos americanos. 2012, 142, p. 113-135

SULLÓN BARRETO, Gleydi. Vasallos y extranjeros. Portugueses en la Lima virreinal, 15701680. Madrid, Universidad Complutense, 2014.

SULLÓN BARRETO, Gleydi. "La presencia femenina entre los inmigrantes portugueses en Lima en el siglo XVII" In Revista complutense de historia de América. 2016, 42, pp. 267-292.

TORRES SANTANA, Elisa. "Lanzarote y Madeira durante la Unión Ibérica a través de la documentación notarial” In III Colóquio Internacional de História da Madeira, Funchal. Centro de Estudos de História do Atlântico, 1993, p. 635-658

TORRES SANTANA, Elisa. “Azoreanos en Lanzarote, 1600-1640” In História das Ilhas Atlânticas. (Arte, Comércio, Demografia, Literatura) Funchal, Centro de Estudos de História do Atlântico, 1997, p. 287-302

VERLINDEN, Charles. "Castellanos, portugueses, italianos y otros pobladores de Canarias a raíz de la conquista. Una cuestión de proporciones" In VI Coloquio de Historia Canario-Americana (1984). Las Palmas de Gran Canaria, Cabildo de Gran Canaria, 1987, p. 11-23.

ZINSSER, Judith P. “Women's History, World History, and the Construction of New Narratives" In Journal of Women's History. 2000, 12, p. 196-206.

Artigo recebido para publicação em 14/09/2020

Artigo aprovado para publicação em 04/03/2021 